\title{
BMJ Global Health Taking digital health innovation to scale in South Africa: ten lessons from MomConnect
}

\author{
Joanne Peter, ${ }^{1}$ Peter Benjamin, ${ }^{2}$ Amnesty Elizabeth LeFevre, ${ }^{3}$ Peter Barron,, 4 \\ Yogan Pillay ${ }^{6}$
}

To cite: Peter J, Benjamin P, LeFevre AE, et al. Taking digital health innovation to scale in South Africa: ten lessons from MomConnect. BMJ Glob Health 2018;3:e000592. doi:10.1136/ bmjgh-2017-000592

Handling editor Seye Abimbola

Received 3 October 2017 Revised 10 December 2017 Accepted 12 December 2017
Check for updates

${ }^{1}$ Global Community Impact, Johnson \& Johnson, Cape Town, South Africa

${ }^{2}$ HealthEnabled, Cape Town, South Africa

${ }^{3}$ International Health, Baltimore, Maryland, USA

${ }^{4}$ School of Public Health, University of the Witwatersrand, South Africa

${ }^{5} \mathrm{HIV}$, TB and MCWH, National Department of Health, Pretoria, South Africa

${ }^{6}$ National Department of Health, Pretoria, South Africa

Correspondence to

Dr Joanne Peter;

jpeter4@its.jnj.com

\section{INTRODUCTION}

In the last decade, hundreds of initiatives that send health information messages through mobile phone networks have emerged in low-resource countries. Most of these have been small in scale, with only five having scaled to $>1$ million beneficiaries: Kilkari and mMitra in India; Healthy Pregnancy, Healthy Baby Text Messaging Service (Wazazi Nipendeni) in Tanzania; Aponjon in Bangladesh; and MomConnect in South Africa. ${ }^{1}{ }^{2}$ Of these programmes, MomConnect has attained the highest population-level coverage, reaching $>60 \%$ of pregnant women attending their first antenatal care appointment in 2017.

In the final editorial of this MomConnect series, we have adapted the framework provided by WHO's 'mHealth Assessment and Planning for Scale' toolkit to summarise lessons learnt across the domains of leadership and partnerships, technology and architecture, content and user engagement, financial health, and monitoring and evaluation. The selected lessons may provide insights to other digital health programmes.

\section{LEADERSHIP AND PARTNERSHIPS}

Lesson 1: High-level government buy-in and leadership is fundamental to any successful national digital health implementation.

MomConnect had its origins in several similar mobile health pilot programmes that were aligned and rationalised by the National Department of Health (NDOH) into a single overarching initiative. It officially launched on 25 August 2014 and within 12 months had enrolled $>494140$ pregnant women. This rapid deployment was made possible by government leadership at the highest level, including the Minister of Health, Dr Aaron Motsoaledi. $\mathrm{He}$ has championed MomConnect from its inception, conducting road shows in all nine provinces in South Africa to stress the importance of its successful implementation, following up on helpdesk utilisation and registration trends during routine provincial visits, and commemorating the achievement of key milestones with public events. MomConnect is under the control of the Deputy Director-General (DDG) in the $\mathrm{NDOH}$ responsible for maternal and child health. The DDG is supported in the day-to-day oversight of MomConnect by a senior technical assistant, who provides strategic guidance on priorities, convenes meetings of the MomConnect partners, oversees reporting on monitoring and evaluation, and supervises helpdesk staff. Provincial employees, usually maternal and child health managers, act as focal points to ensure that programme activities are integrated into regular maternal and child activities. Collectively, this engagement at all levels of the health system has been vital to the success of MomConnect. ${ }^{4}$

Lesson 2: A scaled digital health implementation requires complex multistakeholder partnerships.

The day-to-day operations of MomConnect rely on contributions from $>20$ different partner organisations, including non-profit, for-profit, academic, and donor organisations. The NDOH neither has, nor needs to have, the skills and capacity to run the technical operations of MomConnect. However, the NDOH sets the priorities of MomConnect and provides strategic guidance and clear channels for decision-making and communication to maintain alignment across this range of stakeholders.

\section{TECHNOLOGY AND ARCHITECTURE}

Lesson 3: Open-source software and open standards enable an interoperable system that can grow and expand as technology and requirements change. 
The technical architecture of MomConnect was built following South Africa's Health Normative Standards Framework. ${ }^{5}$ The technical partners developed a roadmap that allowed the MomConnect system to be inclusive, interoperable with other existing health informatics systems, and scalable to national level. ${ }^{67}$

South Africa is implementing a unique patient identifier, which can be captured during the MomConnect registration process and will permit linkages with other platforms such as the National Health Laboratory Service for integration of patient laboratory results and the TIER. Net electronic patient management system for HIV-positive patients on antiretroviral therapy. It is both feasible and straightforward to add other applications to the existing MomConnect platform, making it flexible and extensible for future population groups and use cases (e.g. early childhood development).

Lesson 4: Unstructured Supplementary Service Data (USSD) and Short Message Service (SMS) were the right technology choices when MomConnect launched, but there is urgency to use alternative technology for registration and messaging.

At MomConnect's inception, USSD and SMS were chosen as the preferred channels for registration and messaging, respectively. This was based on their universal accessibility on all handsets, affordability, and familiarity to the target population. South Africa's adult literacy rate is $>90 \%$, making text-based solutions viable. ${ }^{8}$ The rationale of this decision stands, but both technologies have significant limitations. USSD is usually used for short exchanges such as airtime balance enquiries, and in practice has been pushed to its limits by the demands of a longer registration process. Timeouts and network failures are frequent, often requiring multiple user attempts before successful registration occurs. This has resulted in one in four users dropping out of the registration process after initially attempting to register. ${ }^{9}$ MomConnect is considering introducing several alternative ways for users to register to minimise failures. These include a registration application for smartphone users, paper-based data collection with batched data capture when there is network connectivity, the option to call a helpdesk if registration is unsuccessful, and a call-back service to users who attempt but fail to register.

SMS has its own drawbacks in sending messages to women: it is costly at scale and limited to 160 characters. Despite sizeable (up to 50\%) mobile network operator discounts, SMS inventory comprises around $75 \%$ of total MomConnect costs. As smartphone penetration increases, MomConnect needs to deliver messages via internet protocol messaging channels (e.g. WhatsApp) or a custom MomConnect application. This will have the dual effect of reducing costs and allowing for much more flexibility in messaging. However, SMS will likely still be required to reach a proportion of users for some years to come.

Lesson 5: Formal integration with the public health system through facility-based registration, capture of a facility code, and provision of a helpdesk allows MomConnect to generate demand for health services while also collecting data to strengthen supply.

MomConnect is more than a one-way information and behaviour change communication platform. It collects geo-located data on confirmed pregnancies and users' experiences of health service quality through helpdesk engagement. Direct feedback from patients is a powerful medium for the $\mathrm{NDOH}$, with specific complaints generating swift and high-level responses from relevant officials and informing decisions to strengthen service delivery. ${ }^{10}$

MomConnect currently registers $>60 \%$ of all pregnant women attending their first antenatal appointment. This is the highest coverage of any maternal messaging programme globally. For this system to work effectively, registration needs to be further formalised as part of the standard protocol for the first antenatal appointment so that it happens routinely for all women.

\section{CONTENT AND USER ENGAGEMENT}

Lesson 6: It is possible to write evidence-based messages that address the most important identified perinatal and child health problems in South Africa in language that women can understand.

MomConnect's twice-weekly SMS messages are linked to the gestational age of the fetus or age of the infant. Alongside core health information, they provide parenting support and information on fetal development to build trust and an emotional connection to the service.

Qualitative research has shown that users are enthusiastic about the messages, stating that the information is both useful and empowering. Some users save the messages to use as a resource or to share with others. ${ }^{11}$

As technology improves, messaging can become more conversational and individualised, with increased tailoring based on user responses. Group discussion could also be considered.

Lesson 7: SMS messages increase utilisation of the helpdesk, but as demand increases, mechanisms to streamline and improve helpdesk responses must be explored.

The helpdesk was used by $8 \%$ of MomConnect users who overwhelmingly $(79 \%)$ ask questions about health topics. ${ }^{12}$ The number of incoming helpdesk queries is markedly higher on days when outgoing SMS messages are sent, illustrating the positive interplay between 'push' and 'pull' channels of communication in driving user engagement. It is possible to use an automated classification system to identify and triage messages of interest (e.g. women who write about disrespect and abuse in the health system). ${ }^{13}$ While further work is needed to refine and train the automated classifier for use in other content areas, this offers much potential for improving and expanding helpdesk efficiencies and capabilities with minimal added human resources. 


\section{FINANCIAL HEALTH}

Lesson 8: Large-scale mobile health programmes require longterm commitment and earmarked funding for core functions and innovation.

The implementation of MomConnect has been a multimillion dollar endeavour. Even in a steady state, it requires ongoing investment to maintain the technical platform, support the costs of mobile inventory, provide refresher training to health workers, and revise the content when necessary. Moreover, the programme cannot remain static, but requires constant innovation to keep abreast of changes in technology, the national health system, and the needs of users.

Maternal messaging programmes focusing exclusively on demand generation may find it challenging to secure ongoing funding as there is still only limited evidence demonstrating a clear relationship between messaging and increased uptake of maternal and child health services. ${ }^{9}$ MomConnect has received substantial upfront donor investment and a government commitment to fund the core elements of the programme because it has been designed to strengthen both the demand and supply side of service delivery. It will likely always require some degree of external funding to pay for innovation with its associated risks.

\section{MONITORING AND EVALUATION}

Lesson 9: Implementation of maternal messaging programmes should be monitored throughout the complete user journey to identify points of failure from the first contact at registration through to receipt of intended messages.

MomConnect had monitoring in place to track successful registrations and any subsequent user opt-outs. These data were captured in weekly reports, sent to the senior leadership of the Department of Health in each province, and regularly discussed. However, the shortcomings of USSD were not quantified for $>2$ years after the launch of the programme, when a detailed analysis focused further upstream on the percentage of users attending the first antenatal appointment that attempted, but failed to register. Similarly, it is insufficient to assume that any user who has successfully registered continues to receive the full suite of messages as intended. Changes in cellphone numbers are a concern, and data on message delivery success and reasons for non-delivery must be monitored. Procedures for removing inactive registered users will save programme costs, while concurrent efforts are needed to accommodate changes to mobile phone numbers to minimise attrition.

MomConnect data were housed by a range of stakeholders necessitating linkages across multiple data sets. Retrospective attempts to acquire data for monitoring required advanced database engineering skills to transform and normalise the data into a usable format. Monitoring plans should have been clearly articulated at the outset of the programme when the technical platform was still being designed, with mapping of core monitoring indicators to their accompanying data elements and data sources.

Lesson 10: mHealth programmes should consider evaluation from the outset, including collection of baseline data prior to implementation.

Despite a rigorous monitoring component, MomConnect's summative evaluation was limited in its ability to link programme activities and message exposure to increased adoption of healthy behaviours and improved maternal and child health outcomes. There is a trade-off between going to scale rapidly and collecting adequate baseline and subsequent information for rigorous outcome evaluation. MomConnect was rolled out with national coverage before baseline data were collected. The quantitative portion of MomConnect's evaluation assumed an observational design, conducting exit interviews with pregnant and postpartum women attending services in the public sector. The limitations of this research design have made it difficult to demonstrate the benefits of the programme versus other health interventions competing for resources. Future programmes should prioritise collection of baseline or comparator data to generate evidence on the effectiveness of the programme to justify ongoing investment.

\section{CONCLUSION}

MomConnect is the only mobile health programme globally to have reached $>60 \%$ coverage of all pregnant women nationally, with 1.7 million subscribers registered since its launch in 2014. This achievement has been hard fought. It reflects strong government leadership, support from multilateral and bilateral organisations, academic and technology partners, and most importantly the efforts, enthusiasm and engagement of health workers and pregnant and postpartum women throughout South Africa. Though many improvements can be made, MomConnect represents a significant starting point with the ability to add new users, features and links to other health services and databases as technology advances and smartphone access increases.

Acknowledgements The support provided by John Snow, Inc. (JSI) in the President's Emergency Plan for AIDS Relief (PEPFAR) and United States Agency for International Development (USAID)-funded MEASURE Evaluation Strategic Information for South Africa (MEval-SIFSA) project to enable this publication is acknowledged with gratitude.

Contributors JP created the first draft. PB, AELF, PB and YP provided comments and edits. All authors reviewed the final submission.

Competing interests JP is an employee of Johnson \& Johnson, one of the funders of MomConnect.

Provenance and peer review Not commissioned; internally peer reviewed.

Open Access This is an Open Access article distributed in accordance with the Creative Commons Attribution Non Commercial (CC BY-NC 4.0) license, which permits others to distribute, remix, adapt, build upon this work non-commercially, and license their derivative works on different terms, provided the original work is properly cited and the use is non-commercial. See: http://creativecommons.org/ licenses/by-nc/4.0/

(C) Article author(s) (or their employer(s) unless otherwise stated in the text of the article) 2018. All rights reserved. No commercial use is permitted unless otherwise expressly granted. 


\section{REFERENCES}

1. USAID. mHealth Compendium. Special edition 2016: Reaching Scale. http://21028-presscdn.pagely.netdna-cdn.com/wp-content/ uploads/2016/08/mHealth-Compendium-Special-Edition-2016Reaching-Scale-.pdf (accessed 13 Sep 2017).

2. TTC Mobile. Healthy pregnancy, healthy baby project. https:// ttcmobile.com/portfolio/healthy-pregnancy-healthy-baby/ (accessed 13 Sep 2017).

3. World Health Organization. The MAPS toolkit: $m$ Health assessment and planning for scale. Geneva: World Health Organization, 2015. (accessed 25 Aug 2017).

4. Barron P, Peter J, LeFevre AE, et al. Mobile health messaging service and helpdesk for South African mothers (MomConnect): history, successes and challenges. BMJ Glob Health 2018;3(Suppl 2):e000559.

5. Minister of Health. Government notice no. 353: National Health Normative Standards framework for interoperability in eHealth. 2014. Government Gazette No. 37624 http://www.gov.za/sites/www.gov. za/files/37624_ gon353.pdf (accessed 29 Jun 2017).

6. Seebregts C, Barron P, Tanna G, et al. MomConnect : an exemplar implementation of the Health Normative Standards Framework in South Africa. In: Padarath A, King J, Mackie E, Casciola J, eds. South African Health Review 2016. Durban: Health Systems Trust, 2016
7. Seebregts C, Dane P, Parsons AN, et al. Designing for scale: optimising the health information system architecture for mobile maternal health messaging in South Africa (MomConnect). BMJ Glob Health 2018;3(Suppl 2):e000563.

8. United Nations Educational, Scientific, and Cultural Organization (UNESCO) Institute for Statistics. eAtlas of literacy. http://tellmaps com/uis/literacy/\#!/tellmap/-1003531175 (accessed 28 Aug 2017).

9. LeFevre AE, Dane P, Copley C, et al. Unpacking the performance of a mobile health information messaging program for mothers (MomConnect) in South Africa: evidence on program reach and messaging exposure. BMJ Glob Health 2018;3(Suppl 2):e000583.

10. Barron P, Pillay $Y$, Fernandes $A$, et al. The MomConnect mHealth initiative in South Africa: early impact on the supply side of $\mathrm{MCH}$ services. J Public Health Policy 2016;37(Suppl 2):201-12.

11. Skinner D, Delobelle $P$, Pappin $M$, et al. User assessments and the use of information from MomConnect, a mobile phone text-based information service, by pregnant women and new mothers in South Africa. BMJ Glob Health 2018;3(Suppl 2):e000561.

12. Xiong $X$, Kamunyori J, Sebidi J. The MomConnect helpdesk: how an interactive mobile messaging programme is used by mothers in South Africa. BMJ Glob Health 2018;3(Suppl 2):e000578.

13. Engelhard M, Copley C, Watson J, et al. Optimising mHealth helpdesk responsiveness in South Africa: towards automated message triage. BMJ Glob Health 2018;3(Suppl 2):e000567. 